\title{
Short-Term Safety Evaluation of a Multi-Pressure Dial: A Prospective, Open-label, Non-randomized Study
}

\author{
Vance M. Thompson - Tanner J. Ferguson (D) - Iqbal Ike K. Ahmed • \\ Thomas W. Samuelson · Russell J. Swan • Mitch Ibach • \\ John P. Berdahl
}

Received: February 18, 2019 / Published online: March 27, 2019

(C) The Author(s) 2019

\section{ABSTRACT}

Introduction: Prospective, open-label, non-randomized, single site study to assess the safety and tolerability of a multi-pressure dial.

Methods: 30 healthy subjects received application of negative pressure $(-15 \mathrm{mmHg})$ in one eye for 30 minutes and ambient atmospheric pressure in the contralateral eye. To evaluate safety, the primary outcome measures included best corrected visual acuity (BCVA), intraocular pressure (IOP) changes from baseline, slit-lamp and dilated fundus examination findings, and rate of adverse events. Exploratory analyses included in this study also evaluated changes from baseline measurement in tear break-up

Enhanced Digital Features To view enhanced digital features for this article go to https://doi.org/10.6084/ m9.figshare.7825061.

V. M. Thompson · T. J. Ferguson $(\bowtie) \cdot$ M. Ibach · J. P. Berdahl

Vance Thompson Vision, Sioux Falls, SD, USA

e-mail: tannerferg@gmail.com

I. I. K. Ahmed

Prism Eye Institute, Mississauga, Ontario, Canada

T. W. Samuelson

Minnesota Eye Consultants, Minneapolis, MN, USA

R. J. Swan

Vance Thompson Vision, Bozeman, SD, USA time (TBUT) and RNFL thickness measured by OCT. In addition to baseline screening, subjects underwent testing and negative pressure application on day 0 and returned 1 week after the initial visit for clinical testing. The follow-up visit did not include use of the multi-pressure dial (MPD) but repeated baseline testing and evaluated for adverse events.

Results: No adverse events were reported in the study. After short-term wear of the MPD on day 0 , there was a minimal but statistically significant increase of half a line (LogMAR) in BCDVA for study eyes; this increase was not present at 1 week. There were no observed changes in cupdisk ratio and TBUT 1 week after the initial testing. There was a statistically significant pressure reduction in both study and fellow eyes at 1 week following the study, but clinical significance has yet to be determined.

Conclusion: The MPD, which consists of a pair of goggles connected to a handheld, programmable pump, was well tolerated by subjects enrolled in the study. Key safety parameters remained stable after short-term exposure. The favorable safety results of this study support the safety profile of the MPD and promote further investigation of the device as a potential treatment of glaucoma.

Funding: Equinox Ophthalmic, Inc. (Newport Beach, CA).

Keywords: Glaucoma treatment; Multipressure dial; Multi-pressure glaucoma 
management; Normal-tension glaucoma; Openangle glaucoma

\section{INTRODUCTION}

Glaucoma is the second most common cause of blindness worldwide [1]. Because intraocular pressure (IOP) remains the only modifiable risk factor, treatment strategies are primarily aimed at lowering intraocular pressure [2]. However, a significant number of patients still progress despite achieving a "target" IOP value considered in the normal range [3]. The current treatment options for lowering pressure include topical medications, laser procedures and surgery. While significant advancement has occurred in this arena, these options remain imperfect and clinicians caring for glaucoma patients continue to seek better options. Currently, there are no treatment options for glaucoma that are non-laser, non-pharmacologic and non-surgical.

In this study, we utilize the multi-pressure dial, or MPD (Equinox Ophthalmic, Inc., Sioux Falls, SD), which consists of a pair of goggles that separately encloses the periorbital areas of each eye, with each eye connected individually to a pressure-modulating pump. Target negative pressure is programmed into the pump, and with proper fit of the goggles, negative pressure within the microenvironment of the googles is established, which results in a corresponding and instantaneous lowering of IOP [4].

The goal of this study is to evaluate the short-term safety and tolerability of the MPD. We anticipate that the MPD will result in a safe, predictable adjustment of IOP, with safety and tolerability outcomes providing a foundation for larger, longer term studies to further characterize the use of the device as a glaucoma treatment.

\section{METHODS}

Thirty subjects participated in this prospective, open-label, pilot study. Thirty healthy right (OD) eyes of each subject received treatment with negative pressure, while the contralateral eyes served as the control. Demographic characteristics of the study population are shown in Table 1 . This study was approved by the Aspire IRB (Santee, CA) and was performed at a single site (Sioux Falls, SD). All procedures conducted were in accordance with the Aspire IRB and the 1964 Helsinki Declaration and its later amendments or comparable ethical standards. Informed consent was obtained for each subject prior to the study. This study was approved and designated as a non-significant risk (NSR) clinical study by an IRB and did not require clinical trial registration.

All subjects underwent a baseline ophthalmologic examination. The examination included the following diagnostic tests: bestcorrected distance visual acuity (BCDVA), cupdisk ratio (CDR), tear break-up time (TBUT), OCT of the retinal nerve fiber layer (RNFL) and intraocular pressure (IOP). IOP measurement was obtained via Goldmann applanation tonometry (GAT). In addition, a comprehensive slit-lamp and dilated fundus examination were performed.

A summary of the visit schedule with the diagnostic testing and when they were performed is included in Table 2. The visits included screening, day 0 and day 7. Screening and day 0 (testing) may be performed on the same day.

Table 1 Preoperative characteristics

\begin{tabular}{ll}
\hline Parameter & \\
\hline Age, years (mean, SD) & $31.60 \pm 10.00$ \\
Gender (M/F) & $24 \mathrm{~F} / 6 \mathrm{M}$ \\
Ethnicity & Non-Hispanic or Latino/White \\
BCVA (mean $\pm \mathrm{SD})$ & \\
Study eye & $-0.07 \pm 0.07$ \\
Fellow eye & $-0.05 \pm 0.07$ \\
Cup-disc ratio (mean \pm SD) \\
Study eye & $0.33 \pm 0.12$ \\
Fellow eye & $0.33 \pm 0.13$ \\
\hline
\end{tabular}

$B C V A$ best-corrected visual acuity 
Table 2 This table demonstrates the visit schedule for each subject and the tests included

\begin{tabular}{|c|c|c|c|c|}
\hline \multicolumn{5}{|l|}{ Visit schedule } \\
\hline \multirow{2}{*}{ Procedure } & \multicolumn{3}{|l|}{ Screening/day 0} & \multirow[t]{2}{*}{ Day 7} \\
\hline & Prior to MPD & During MPD & $\overline{\text { After MPD }}$ & \\
\hline Informed consent & $\mathrm{x}$ & & & \\
\hline Urine pregnancy test & $\mathrm{x}$ & & & \\
\hline OCT of RNFL & $\mathrm{x}$ & $x^{*}$ & $\mathrm{x}$ & $\mathrm{x}$ \\
\hline Tear break-up time (TBUT) & $\mathrm{x}$ & & $\mathrm{x}$ & $\mathrm{x}$ \\
\hline Keratometry & $\mathrm{x}$ & & $\mathrm{x}$ & $\mathrm{x}$ \\
\hline Axial length & $\mathrm{x}$ & & $\mathrm{x}$ & $\mathrm{x}$ \\
\hline MRX & $\mathrm{x}$ & & $\mathrm{x}$ & $\mathrm{x}$ \\
\hline BCDVA & $\mathrm{x}$ & & $\mathrm{x}$ & $\mathrm{x}$ \\
\hline SLE & $\mathrm{x}$ & & $\mathrm{x}$ & $\mathrm{x}$ \\
\hline DFE & $\mathrm{x}$ & $x^{* *}$ & $\mathrm{x}$ & $\mathrm{x}$ \\
\hline IOP & $\mathrm{x}$ & & $\mathrm{x}$ & $\mathrm{x}$ \\
\hline
\end{tabular}

Note that the negative pressure application only occurred on day 0

$M R X$ manifest refraction, $B C D V A$ best-corrected distance visual acuity, SLE slit-lamp examination, DFE dilated fundus examination, $I O P$ intraocular pressure, $M P D$ multi-pressure dial

*During application of negative pressure, 2 OCT images were obtained of each eye at 5 and $25 \mathrm{~min}$

${ }^{* *}$ DFE will be completed 15 min into application of negative pressure

Key inclusion criteria were subjects $\geq 18$ years of age with dilated IOP between 10-25 mmHg. Exclusion criteria included the presence or history of any eye disease or condition that could interfere with the assessment of the study results or subject safety. Subjects with glaucoma in either eye, macular degeneration, retinal detachment or other fundus findings that could inhibit visualization of the retina in either eye, eyelid edema or conjunctival chemosis in either eye, history of corneal transplant in either eye or history of allergy to any of the testing materials (e.g., silicone) were excluded. Women who were pregnant or lactating during the time of the study were also excluded.

\section{Study Design}

The right eyes of subjects who passed screening were enrolled into the study and fitted with the
MPD (Fig. 1) to ensure a secure and comfortable fit. All baseline testing occurred prior to wear of the MPD. The initial IOP measurements occurred prior to placing the MPD and were repeated immediately after removal. After the MPD was adjusted to fit securely and comfortably, negative pressure was gradually applied in the study eye until the target value, $-15 \mathrm{mmHg}$, was achieved. When the target value was reached and verified with a manometer, this was considered time point 0 and the patient wore the system for $30 \mathrm{~min}$. After $5 \mathrm{~min}$ and $25 \mathrm{~min}$ of wear time, 4 OCT images of the RNFL were obtained. At the 15-min mark, a dilated fundus examination was performed. After 30 min of wear time, the pump applying negative pressure was slowly released and the MPD was removed. "Day 0" will be used throughout this report to denote the initial evaluation after removal of the MPD. After removal of the device (day 0), the baseline 


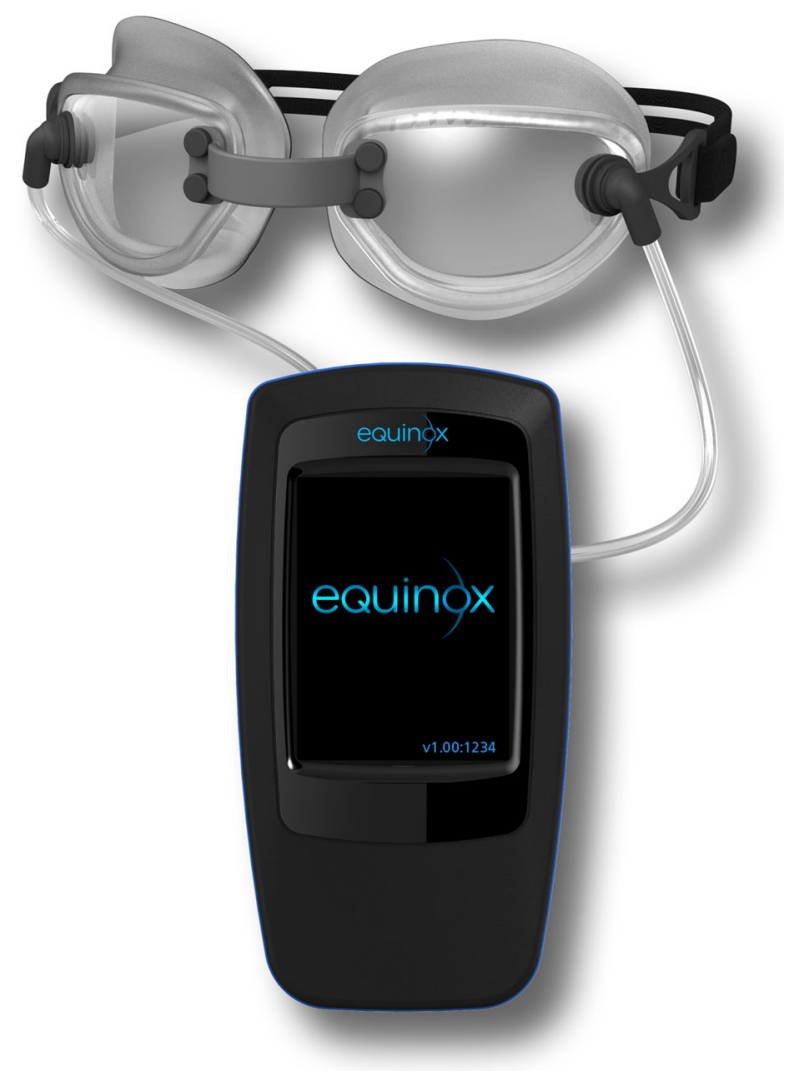

Fig. 1 This figure demonstrates the multi-pressure dial (MPD), which includes the goggles connected to a handheld pressure-modulating pump

testing was immediately repeated. Subjects returned within 1 week (6-8 days) to repeat testing.

At the 1-week visit, baseline testing was repeated and subjects were also assessed for adverse events that may have occurred in the 7 days following the use of the MPD. If adverse events were observed, patients were appropriately managed and treated for resolution of the adverse event.

\section{Statistical Analysis}

The mean change in IOP from baseline to day 0 (immediately following study period) and baseline to 1 week was analyzed by a one-sample Student's $t$-test where the data are the difference from the baseline value with the null hypothesis being the calculated mean difference is equal to zero. The change in BCVA from baseline to day 0 and baseline to 1 week was analyzed in an identical fashion. An $\alpha$ level of 0.05 was considered statistically significant. All of the statistical analysis was conducted using $\mathrm{SAS}{ }^{\circledR}$ software.

\section{RESULTS}

\section{Subject Demographics}

This study evaluated 30 right (OD) eyes from 30 subjects. The 30 left (OS) eyes were used as controls. The mean age of the subjects was 31.6 \pm 10.0 . Of the 30 subjects, 24 were female. All subjects included in the study were non-Hispanic or Latino/White. This demographic information is included with other preoperative characteristics in Table 1.

The IOP results are demonstrated in Fig. 2. Prior to wear of the MPD, the mean baseline IOP of the study $(n=30)$ and fellow eyes $(n=30)$ was $15.40 \pm 2.56 \mathrm{mmHg}$ and $14.73 \pm$ $2.33 \mathrm{mmHg}$, respectively. There was not a statistically significant difference between groups at baseline $(p=0.30), 30 \mathrm{~min}(p=0.31)$ and 1 week $(p=0.77)$. After $30 \mathrm{~min}$ of wearing the MPD, the mean IOP in the study eye was 14.88 $\pm 2.62 \mathrm{mmHg}$. In the fellow eye (which did not receive negative pressure), the mean IOP was $14.22 \pm 2.46$. At the 1 -week visit, IOP in the study eyes was $14.13 \pm 2.94 \mathrm{mmHg}$, indicating a $1.27 \mathrm{mmHg}$ mean reduction in pressure from baseline, which was statistically significant $(p<0.05)$. In the fellow eyes, the mean reduction in pressure was $0.82 \mathrm{mmHg}(p<0.05)$. There were no IOP spikes observed in either eye immediately following wear of the MPD or at the 1-week follow-up visit.

At baseline, the mean LogMAR BCDVA of the study and fellow eyes of the subjects was $-0.07 \pm 0.06$ and $-0.05 \pm 0.07$, respectively. After the study period, there was a minimal but statistically significant increase of half a line from $-0.07 \pm 0.06$ to $-0.02 \pm 0.09$ in BCDVA for the study eyes on day $0(p<0.05)$. At 1 week, this increase was no longer statistically significant as BCDVA values returned closer to baseline $(-0.06 \pm 0.06)$. The BCDVA and CDR 


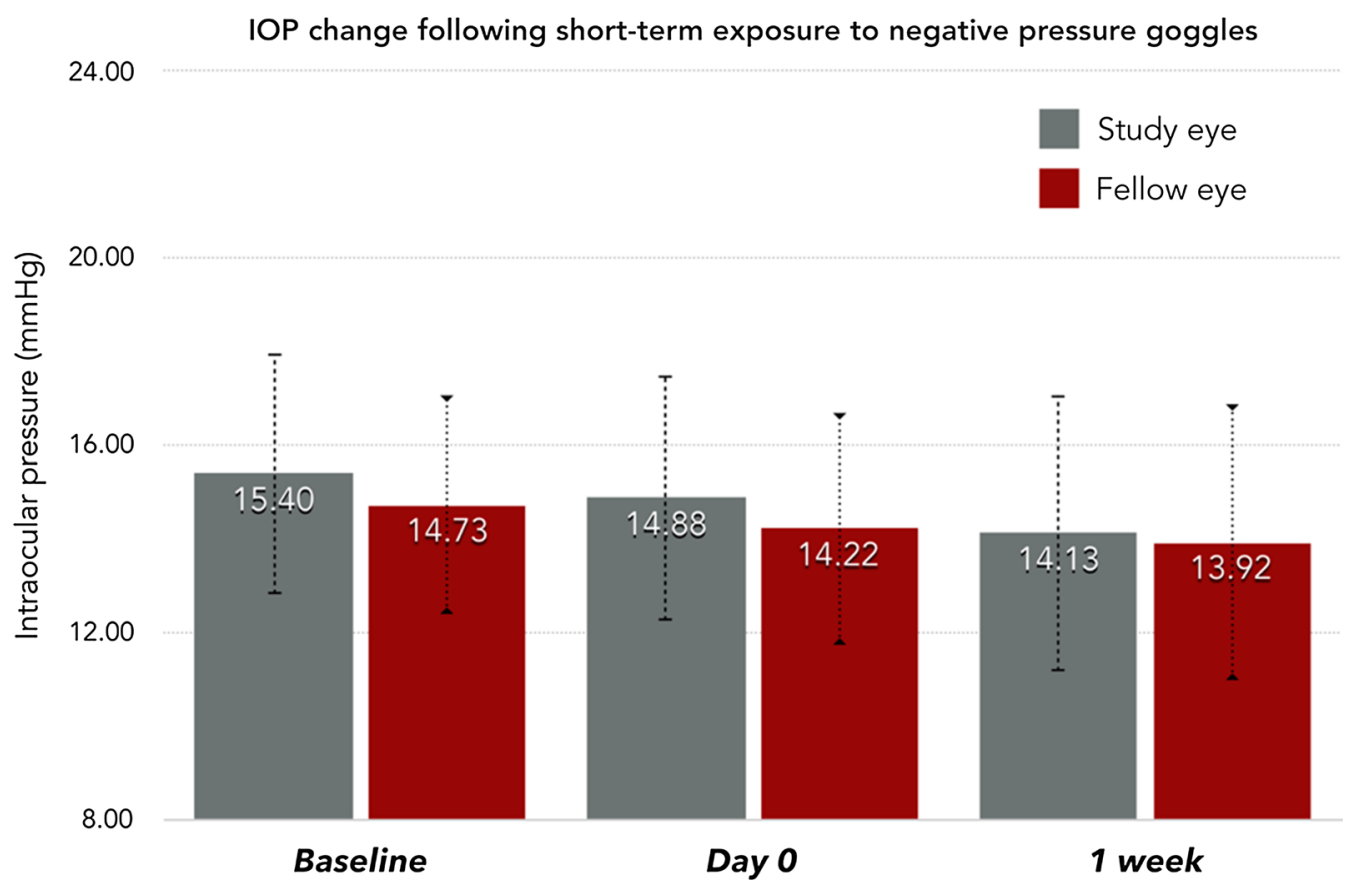

Fig. 2 This graph demonstrates the mean IOP for the study and fellow eyes at each time point in the study visit schedule. The error bars indicate standard deviation

changes after application of negative pressure are shown in Table 3.

There were no observed differences from baseline in either the study or fellow eye in the slit-lamp or dilated fundus examination after wearing the MPD. No new or abnormal slitlamp or dilated fundus examination findings were noted at the follow-up visit 1 week later. At baseline, the mean CDR of the study and fellow eyes was $0.33 \pm 0.12$ and $0.33 \pm 0.13$, respectively. The CDR values initially after removal of the MPD and at 1 week were unchanged. The TBUT values obtained after removal of the MPD

Table 3 Changes in cup-disc ratio and BCVA following short-term exposure to negative pressure goggles

\begin{tabular}{lccc}
\hline Parameter & Day 0 & 1 week & P value \\
\hline Cup-disc ratio (mean, SD) & $0.33 \pm 0.12$ & $0.33 \pm 1.12$ & $>0.05$ \\
Study eye & & & $>0.33 \pm 0.12$ \\
Cup-disc ratio (mean, SD) & $0.33 \pm 0.13$ & \\
Control eye & & $-0.06 \pm 0.06$ \\
BCVA (mean, SD) & $-0.02 \pm 0.09$ & \\
Study eye & & $-0.05 \pm 0.08$ & $>0.05$ \\
BCVA (mean, SD) & $-0.03 \pm 0.09$ & \\
Control eye & &
\end{tabular}

Day 0 depicts the measurements obtained immediately after the 30-min negative pressure application $B C V A$ best-corrected visual acuity, $S D$ standard deviation 
and after 1 week were unchanged from baseline in both the study and fellow eye.

This study did not utilize a subjective questionnaire to assess the tolerability of the MPD. However, subjects were asked to report discomfort and/or issues with tolerability. There were no such reports, all subjects successfully tolerated the 30-min test duration. No adverse events were reported during this study.

\section{DISCUSSION}

The current treatment options for open-angle glaucoma are primarily aimed at lowering intraocular pressure. The emergence of MIGS (minimally invasive glaucoma surgery) has revolutionized the management of glaucoma, and newer medical therapies have expanded the armamentarium for providers with improved safety and efficacy [5-7]. However, despite the emergence of new treatment options, these new options are imperfect and still carry risk. Currently, there are no treatment options that are non-laser, non-pharmacologic and noninvasive.

While intraocular pressure (IOP)-lowering strategies have been the mainstay of glaucoma treatment, a significant number of patients still progress despite a decrease in IOP following treatment [3]. One possible explanation for this is that optic nerve damage occurs because of an imbalance between IOP and cerebrospinal fluid pressure (CSFp) posterior to the optic nerve. This is also known as the translaminar pressure difference (TLPD). When a person dives deep below sea level and experiences a significant increase in atmospheric pressure, this pressure acts on the entire body, including the CSFp and IOP, maintaining the TLPD. This explanation is supported by previous studies that suggest TLPD may play a significant role in the pathogenesis of glaucoma [8-11].

This present report evaluated the safety of a pair of goggles in the periorbital region attached to an intraocular pressure-adjusting pump, collectively known as the multi-pressure dial (MPD). The MPD establishes a microenvironment and produces a decrease in atmospheric pressure contacting the eye with the application of a negative pressure pump. This decrease in atmospheric pressure applied to the eye generates a corresponding instantaneous decrease in IOP. By lowering the pressure in the eye relative to other tissues in the body (e.g., blood pressure, CSFp) with the MPD, this may facilitate the decoupling of IOP from the CSFp. A graphic of this concept is shown in Fig. 3.

A poster presentation in 2019 [4] demonstrated the IOP-lowering capabilities of the MPD at various negative pressure settings in healthy subjects. The results of this randomized, controlled study also showed that IOP reduction can be produced in a titratable fashion, even in normotensive eyes with the MPD. The MPD system evaluated in this study is similar to what was investigated in the aforementioned presentation.

The results of this study suggest that the MPD system is safe and tolerable for patients. No patients in this study endured any adverse events, and all patients were able to safely and comfortably wear the MPD for the duration of the study period. There were no observed poststudy IOP spikes. The mean IOP values were actually lower 1 week after the study period in both the study and fellow (control) eye, and the difference was statistically significant. Although the modest IOP reduction observed at 1 week was statistically significant, it could be unrelated to treatment related to the device's IOPlowering ability and influenced by other factors such as IOP variability or diurnal variation.

This study is not without limitations. The sample size was small; controlled studies with longer duration and larger sample sizes would further elucidate the safety and efficacy of this device. Moreover, it is difficult to discern what negative effects, if any, the MPD may have over an extended treatment period. However, despite the limitations, we think the results of this preliminary study are meaningful and will promote further research of the device. Moreover, the initial results of this study are very promising; it was well tolerated by subjects, and the safety parameters used in this study demonstrated no indication that the MPD is not safe for use.

One potential limitation is that the vacuum pump or application of negative pressure by the 


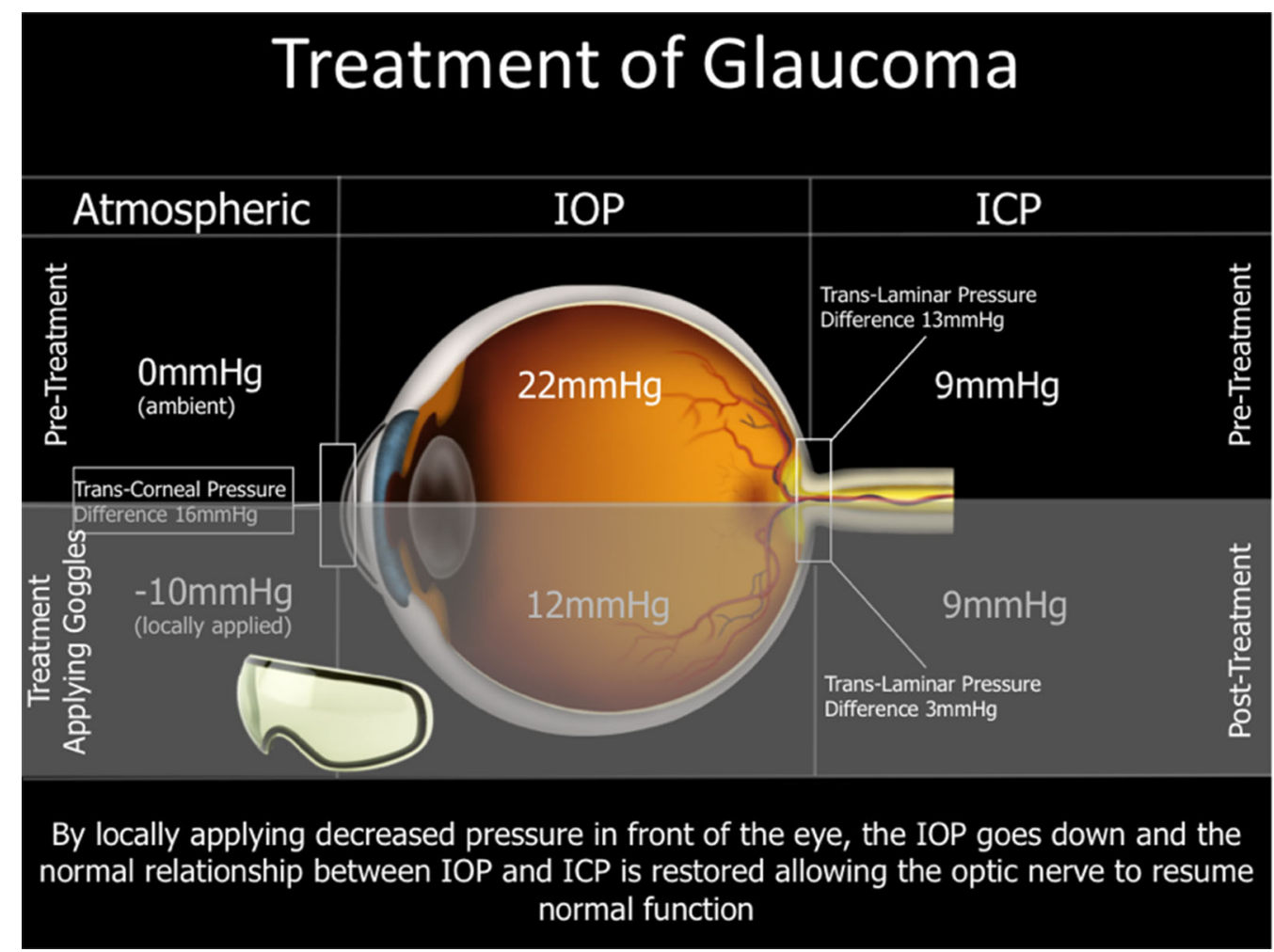

Fig. 3 This figure illustrates how negative pressure locally applied by the goggles can lower intraocular pressure and restore the normal relationship between IOP and ICP

MPD in the microenvironment could presumably dry out the ocular surface and trigger dry eye symptoms and possibly changes in TBUT values. However, in this study, all 30 patients had TBUT values unchanged from baseline in both the fellow and study eyes upon removal of the MPD as well as after 1 week. Moreover, there were no reports of dryness or irritation while wearing the device. Furthermore, none of the study subjects reported symptoms in the 7 days following wear of the MPD at their 1-week follow-up assessment. Given the tight seal of the MPD and the findings of this small study, it is possible the device may provide a secondary benefit of dry eye relief by acting as a moisture chamber, similar to what is performed in patients at risk of exposure keratopathy. However, further research would be valuable in exploring and validating this proposed benefit of dry eye relief.

At this point, it is unclear what, if any, effect the MPD's negative atmospheric pressure application to the anterior orbital rim has on episcleral venous pressure or aqueous flow transversing the trabecular meshwork. This was a short-term study to evaluate preliminary safety and tolerability prior to undertaking longer term studies to further characterize the safety profile. Future studies investigating longer term wear may convey more insight on the MPD's impact on factors proposed to contribute to the pathogenesis of glaucoma-episcleral venous pressure, trabecular meshwork resistance, etc.

\section{CONCLUSION}

Utilizing a localized vacuum to carefully titrate the pressure inside the eye with the multipressure dial represents a new approach to treatment known as multi-pressure glaucoma management. This individualized strategy has potentially important therapeutic implications for glaucoma. The favorable findings in this 
report support the short-term safety of the device and promote further research of the MPD as a potential non-pharmacologic, non-surgical treatment of glaucoma.

\section{ACKNOWLEDGEMENTS}

We thank the study participants for their participation in the study.

Funding. This study was sponsored by Equinox Ophthalmic, Inc. (Sioux Falls, SD). Article processing charges were funded by Equinox Ophthalmic, Inc. All authors had complete access to the study data and assume full responsibility for the integrity of the data and the accuracy of the data analysis.

Authorship. All named authors meet the International Committee of Medical Journal Editors (ICMJE) criteria for authorship for this article, take responsibility for the integrity of the work as a whole, and have given their approval for this version to be published.

Disclosures. Dr. Berdahl is the founder and CEO of Equinox Ophthalmic, Inc. Vance $M$. Thompson is a consultant for Equinox Ophthalmic, Inc. Tanner J. Ferguson is a consultant for Equinox Ophthalmic, Inc. Iqbal Ike Ahmed is a consultant for Equinox Ophthalmic, Inc. Thomas W. Samuelson is a consultant for Equinox Ophthalmic, Inc. Russell J. Swan is a consultant for Equinox Ophthalmic, Inc. Mitch Ibach is a consultant for Equinox Ophthalmic, Inc.

Compliance with ethics guidelines. All procedures conducted were in accordance with the Aspire IRB and the 1964 Helsinki Declaration and its later amendments or comparable ethical standards. Informed consent was obtained for each subject prior to the study. This study was approved and designated as a non-significant risk (NSR) clinical study by an IRB and did not require clinical trial registration.

Data Availability. The data set collected and analyzed for this present study is available from the corresponding author per reasonable request.

Open Access. This article is distributed under the terms of the Creative Commons Attribution-NonCommercial 4.0 International License (http://creativecommons.org/licenses/ by-nc/4.0/), which permits any noncommercial use, distribution, and reproduction in any medium, provided you give appropriate credit to the original author(s) and the source, provide a link to the Creative Commons license, and indicate if changes were made.

\section{REFERENCES}

1. Kingman S. Glaucoma is second leading cause of blindness globally. Bull World Health Organ. 2004;82:887-8. https://doi.org/10.1590/S0042-96 862004001100019 .

2. Tham Y-C, Li X, Wong TY, Quigley HA, Aung T, Cheng C-Y. Global prevalence of glaucoma and projections of glaucoma burden through 2040: a systematic review and meta-analysis. Ophthalmology. 2014;121(11):2081-90. https://doi.org/10. 1016/j.ophtha.2014.05.013.

3. Leske MC, Heijl A, Hussein M, Bengtsson B, Hyman L, Komaroff E. Factors for glaucoma progression and the effect of treatment: the early manifest glaucoma trial. Arch Ophthalmol. 2003;121(1): 48-56. https://doi.org/10.1001/archopht.121.1.48.

4. Swan R, Kahook MY, Samuelson TW, Ahmed IK, Lewis R, Lindstrom R, Radcliffe N, Thompson V, Berdahl JP. Negative Pressure Applied to the Periocular Microenvironment Anterior to the Orbital Rim to Lower Intraocular Pressure. San Francisco, California: American Glaucoma Society; 2019.

5. Saheb H, Ahmed I. Micro-invasive glaucoma surgery: current perspectives and future directions. Curr Opin Ophthalmol. 2012;23(2):96-104.

6. Grace M, Richter ALC. Minimally invasive glaucoma surgery: current status and future prospects. Clin Ophthalmol (Auckland, NZ). 2016;10: 189-206. https://doi.org/10.2147/OPTH.S80490.

7. Weinreb RN, Ong T, Sforzolini BS, Vittitow JL, Singh K, Kaufman PL. A randomised, controlled comparison of latanoprostene bunod and latanoprost $0.005 \%$ in the treatment of ocular hypertension and open angle glaucoma: the VOYAGER 
study. Br J Ophthalmol. 2014;99(6):738-45. https:// doi.org/10.1136/bjophthalmol-2014-305908.

8. Berdahl JP, Allingham RR. Cerebrospinal fluid pressure may play a role in reversal of cupping after glaucoma surgery. Am J Ophthalmol. 2009;148(4): 623-4. https://doi.org/10.1016/j.ajo.2009.06.002 (authorreply624-5).

9. Berdahl JP, Allingham RR, Johnson DH. Cerebrospinal fluid pressure is decreased in primary open- angle glaucoma. Ophthalmology. 2008;115(5): 763-8. https://doi.org/10.1016/j.ophtha.2008.01.013.

10. Berdahl JP, Allingham RR. Intracranial pressure and glaucoma. Curr Opin Ophthalmol. 2010;21(2):10611. https://doi.org/10.1097/ICU.0b013e32833651d8.

11. Ren R, Jonas JB, Tian G, et al. Cerebrospinal fluid pressure in glaucoma: a prospective study. Ophthalmology. 2010;117(2):259-66. https://doi.org/ 10.1016/j.ophtha.2009.06.058. 